\title{
Stereotactic body radiation therapy for oligoprogression of metastatic disease from gastrointestinal cancers: A novel approach to extend chemotherapy efficacy
}

\author{
JUSTIN WRAY ${ }^{1 *}$, RANA FAWZI HAWAMDEH ${ }^{2 *}$, NALINI HASIJA ${ }^{2}$, ROI DAGAN ${ }^{3}$, \\ ANAMARIA R. YEUNG ${ }^{1}$, JUDITH L. LIGHTSEY ${ }^{1}$, PAUL OKUNIEFF ${ }^{1,4}$, KAREN C. DAILY ${ }^{2}$, \\ THOMAS J. GEORGE ${ }^{2}$, ROBERT A. ZLOTECKI ${ }^{1}$, JOSE TREVINO ${ }^{4}$ and LONG H. DANG ${ }^{2}$
}

\begin{abstract}
${ }^{1}$ Department of Radiation Oncology; ${ }^{2}$ Division of Hematology and Oncology, Department of Internal Medicine, University of Florida, Gainesville, FL 32610; ${ }^{3}$ University of Florida Health Proton Therapy Institute, Jacksonville, FL 32206; ${ }^{4}$ Department of General Surgery, University of Florida, Gainesville, FL 32610, USA
\end{abstract}

Received April 4, 2015; Accepted June 27, 2016

DOI: $10.3892 / \mathrm{ol} .2016 .5540$

\begin{abstract}
Chemotherapy and targeted therapies are effective palliative options for numerous unresectable or metastatic cancers. However, treatment resistance inevitably develops leading to mortality. In a subset of patients, systemic therapy appears to control the majority of tumors leaving 5 or less to progress, a phenomenon described as oligoprogression. Reasoning that the majority of lesions remain responsive to ongoing systemic chemotherapy, we hypothesized that local treatment of the progressing lesions would confer a benefit. The present study describes the cases of 5 patients whose metastatic disease was largely controlled by chemotherapy. The oligoprogressive lesions $(\leq 5)$ were treated with stereotactic body radiotherapy (SBRT), justifying continued use of an effective systemic regimen. A total of 5 patients with metastatic disease on chemotherapy, with $\leq 5$ progressing lesions amenable to SBRT, were treated with ablative intent. Primary tumor site and histology were as follows: 2 with metastatic colon adenocarcinoma, 2 with metastatic rectal adenocarcinoma and 1 with metastatic pancreatic adenocarcinoma. Imaging was performed prior to SBRT and every 3 months after SBRT. In total, 4 out of the 5 patients achieved disease control for $>7$ months with SBRT, without changing chemotherapy regimen. The median time to chemotherapy change was 9 months, with a median follow-up time of 9 months. The patient who failed to respond developed progressive disease
\end{abstract}

Correspondence to: Dr Robert A. Zlotecki, Department of Radiation Oncology, University of Florida, 2000 SW Archer Road, PO Box 100385, Gainesville, FL 32610, USA

E-mail: zlotera@ufl.edu

${ }^{*}$ Contributed equally

Key words: stereotactic body radiation therapy, chemotherapy, oligoprogression, outcomes outside of the SBRT field at 3 months. In conclusion, the addition of SBRT to chemotherapy is an option for the overall systemic control of oligoprogressive disease.

\section{Introduction}

In patients with metastatic solid tumors treated with systemic chemotherapy, targeted therapy or both, oligoprogression is a relatively new term used to describe the clinical scenario in which a solitary or a few $(\leq 5)$ metastatic tumors progress, while all other sites of disease are stable or responding to the current regimen (1). The concept of oligoprogression is based in the biological principle of branched cancer evolution. Tumor biopsies and cadaveric studies have shown significant genetic heterogeneity between the primary tumor mass and metastatic lesions, and also amongst different metastatic lesions in the same patient (2-4). This genetic heterogeneity is believed to explain the mixed response to systemic therapy often observed in clinical practice.

One of the main factors that restricts the effectiveness of chemotherapy is acquired resistance. Drug resistance is a complex issue, as a number of factors affect drug sensitivity, including drug activation and inactivation, accelerated drug efflux, drug target alterations, processing of drug-induced damage, DNA methylation and apoptotic evasion (5). Chemotherapy-induced genetic events lead to the development of new drug resistance and the standard of care in this clinical scenario is typically to change the systemic therapy. This approach introduces new problems, including added expense, different toxicities, dose adjustments, different supportive care treatments, patient treatment schedule changes and potentially, decreased efficacy.

Stereotactic body radiotherapy (SBRT) is a highly conformal radiation technique that delivers high-dose radiation to a tumor while sparing much of the nearby normal organs in a timely manner and with excellent tolerance. Compared with conventional palliative radiation therapy, which is delivered over 10 to 15 days with the intent to control pain but not disease progression, SBRT is commonly delivered in 
3 to 10 treatments with the intent of disease ablation. There is evidence that SBRT achieves local control rates in excess of $90 \%$. A number of studies also boast long-term disease-free survival and overall survival (6-8). SBRT has become the standard treatment in numerous institutions for oligometastatic disease presentation (1-5 metastases) in coordination with definitive local therapy.

For oligoprogressive cancers, the goal of the present study was to increase the duration until out of field tumor progression, indicating prolonged efficacy of the chemotherapeutic regimen. The primary outcome was to evaluate the time from SBRT to the initiation of subsequent chemotherapy. Finally, the study also hoped to shed light on the hypothesis that SBRT may overcome treatment resistance through sterilization of the few tumor clones that evade otherwise effective systemic therapy.

\section{Patients and methods}

Patients. Patients eligible for treatment presented with metastatic disease in the thorax, liver or adrenals on chemotherapy, with 5 or fewer progressive metastases smaller than $5 \mathrm{~cm}$ in axial diameter. The patients were amenable to SBRT with other sites of disease remaining stable on the current systemic therapy. All patients were enrolled on an institutional review board-approved prospective outcomes tracking protocol in the Department of Radiation Oncology at the University of Florida (Gainesville, FL, USA). All cases were thoroughly reviewed by the Department of Pathology at the University of Florida, and the diagnoses were clear. The majority of the treated lesions were not biopsied beyond the primary diagnosis.

Chemotherapy. Systemic treatment was cancer histology-specific. The details of the individual regimens are presented with the patients' results, including the sequencing of the chemotherapy with SBRT.

Radiation therapy. All patients received SBRT with ablative intent concurrently to each of the progressive metastatic sites. Immobilization occurred in a vacuum cushion. The gross tumor volume (GTV) and internal target volume (ITV) were defined using 4-dimensional (4D) computed tomography (CT) simulation with Pinnacle Software (Phillips, Andover, MA, USA). The GTV was defined in the $50 \%$ phase of the 4D CT and the ITV was expanded to account for breathing motion. There was no clinical target volume expansion and a 5-mm planning target volume (PTV) was isometrically expanded from the ITV. The dose specified to the GTV D95\% ensured that, at minimum, $95 \%$ of the PTV was covered by the $80 \%$ isodose line. Target localization was achieved using on-board image guidance with $\mathrm{kV}$ cone-beam $\mathrm{CT}$ and computer-assisted image registration for each fraction, then confirmed by the treating physician and a medical physicist. Treatment couch repositioning was applied for all translational corrections and rotational corrections were not performed if $<3$ degrees in each axis. Treatment was delivered using conformal arcs or multiple fixed coplanar beams shaped with multileaf collimators. The dose per fraction and total dose were determined using the dose-volume histogram of the organs at risk; individual patient doses are described in the Results section.
Endpoint. The primary endpoint was the progression-free interval, defined as the time from oligoprogression treated with SBRT to the initiation of a different systemic drug or regimen. Disease progression requiring a treatment change was based on radiographic or clinical progression. Patients with dosage adjustments or treatment breaks with re-initiation of the same chemotherapy were not considered to have changed chemotherapy.

\section{Results}

Summary of results. A total of 5 patients were treated with SBRT for oligoprogressive disease and followed until the initiation of a new systemic therapy regimen. The group consisted of 2 patients with colon cancer, 2 with rectal cancer and 1 with pancreatic cancer. Patient characteristics are shown in Table I. At the time of SBRT, 4 patients were on second-line and 1 patient was on third-line chemotherapy. The median time from diagnosis to SBRT was 35 months (range, 11-72 months). The mean and median time to chemotherapy change from first SBRT treatment was 11.7 and 10.6 months, respectively. The median follow-up time was 10 months (range, 3-17 months). During the interval from oligoprogression to chemotherapy change, 2 patients received 2 separate courses of SBRT, while 3 patients were treated with SBRT once. After the second course of SBRT, the time to chemotherapy change was 3 and 5 months in the 2 patients, respectively. All treatments met the aforementioned criteria defined for oligometastatic disease and chemotherapy was held during SBRT. The median time from SBRT to mortality or last follow-up was 10 months (range, 5-27 months) and the time from diagnosis to mortality or last follow-up was 41 months (range, 21-90 months). There were no grade 3 or higher toxicities associated with SBRT per the Common Terminology Criteria for Adverse Events, version 4.0 (http://evs.nci.nih.gov/ftp1/CTCAE/About.html). All patients were staged using the American Joint Committee on Cancer's Cancer Staging Manual, 7th edition (9).

Patient 1. The patient was a 62-year-old male at the time of SBRT and had been diagnosed with stage III (T4N1M0) adenocarcinoma of the pancreas. An exploratory laparotomy was performed 10 months prior to SBRT and unresectable cancer was found invading into the origin of the gastroduodenal artery and proper hepatic artery. A FOLFIRINOX chemotherapy regimen was started consisting of $180 \mathrm{mg} / \mathrm{m}^{2}$ irinotecan, 2,400 mg/m² 5-fluorouracil (5-FU) via continuous intravenous infusion (CIVI) and $85 \mathrm{mg} / \mathrm{m}^{2}$ oxaliplatin, every 2 weeks, and 4 cycles were completed.

Restaging imaging after 3 months showed an unresectable, poorly-defined mass encasing the common hepatic artery. This case was discussed at the University of Florida Gastrointestinal Interdisciplinary tumor board conference (GI TB) with recommendations to continue with systemic chemotherapy for locally advanced pancreatic cancer and withhold radiation therapy until the patient became symptomatic. Due to the disease progression, the patient was started on a new systemic chemotherapy consisting of $1,000 \mathrm{mg} / \mathrm{m}^{2}$ gemcitabine and $125 \mathrm{mg} / \mathrm{m}^{2}$ protein-bound paclitaxel (on days [D]1, 8 and 15) every 28 days. Repeat imaging after 3 cycles showed stable disease except for one ill-defined $1.3-\mathrm{cm}$ liver 


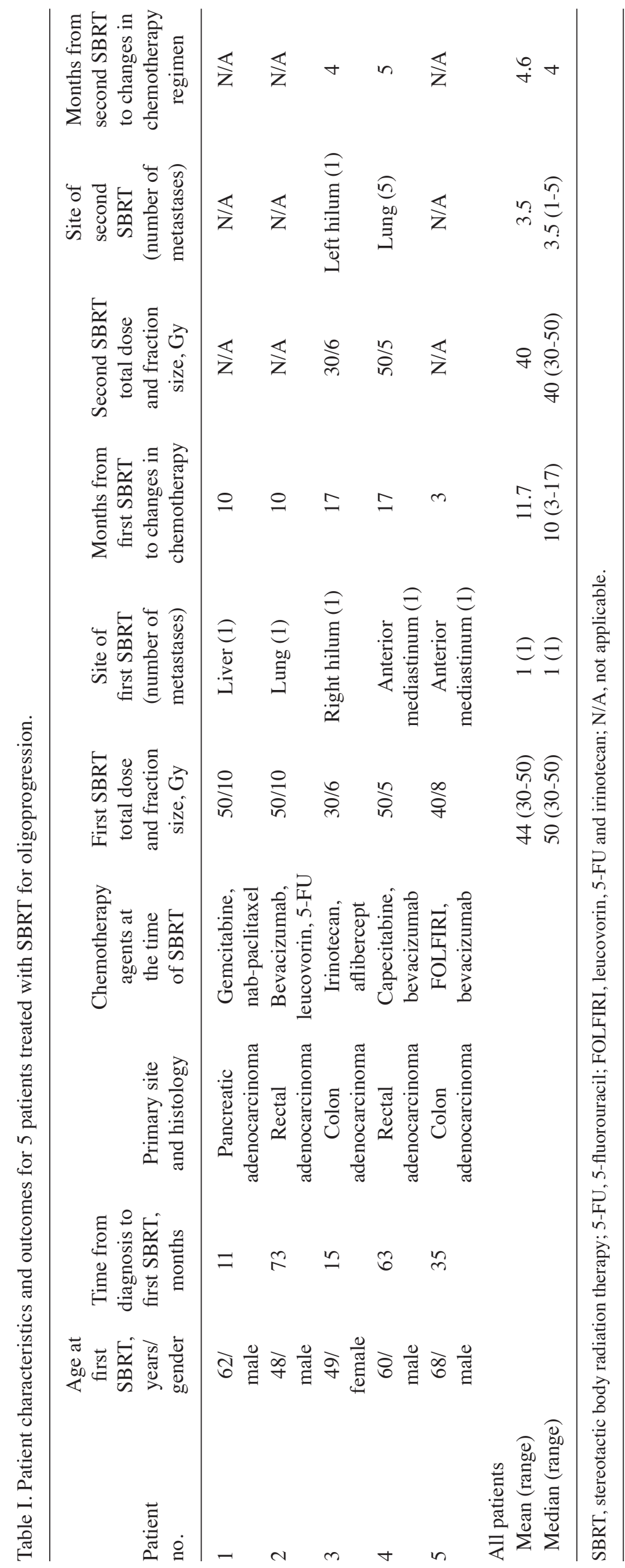



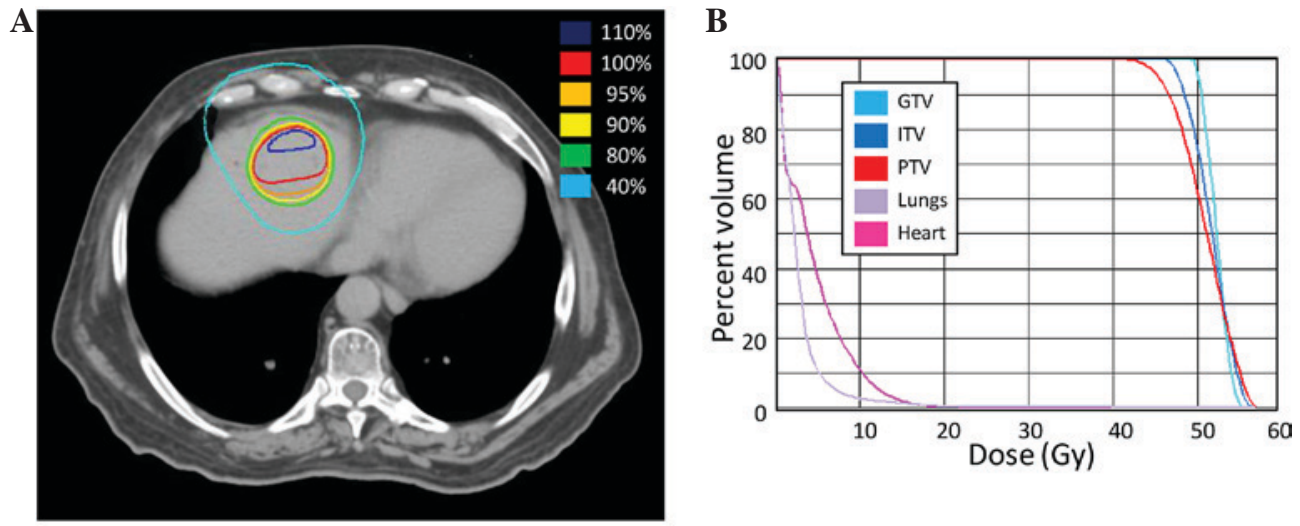

Figure 1. Stereotactic body radiotherapy (SBRT) treatment plan for patient 1. (A) A computed tomography image of the treatment plan with isodose volumes shown. (B) A dose-volume histogram of the SBRT plan with pertinent structures shown. GTV, gross tumor volume; ITV, internal target volume; PTV, planning target volume.

lesion. Chemotherapy was continued, and following cycle 5, imaging showed another 2.2-cm lesion in the left hepatic lobe, with stable disease elsewhere, including the primary site and 1.3 - $\mathrm{cm}$ liver mass.

SBRT to the oligoprogressive liver metastases was delivered at a total dose of 50 Gy over 5 fractions of 10 Gy (Fig. 1). During radiation treatment, chemotherapy was withheld.

Following SBRT, the patient requested a chemotherapy break and has since been off of systemic therapy for 10 months, with stable disease on serial CT scans. Representative imaging is presented in Fig. 2.

Patient 2. The patient was a 48 -year-old male at the time of SBRT and had been diagnosed with stage III (T3N1M0) invasive moderately-differentiated adenocarcinoma of the rectum, with an $8.7 \times 5-\mathrm{cm}$ rectal mass, 6 years prior to SBRT. The patient underwent a diverting colostomy, 6 weeks of concurrent neoadjuvant chemoradiation with 5-FU CIVI D1-5, which was delivered at an external institution, and then a resection that confirmed locally invasive adenocarcinoma. Adjuvant mFOLFOX6 (400 mg/m² 5-FU D1, 2,400 mg/m² 5-FU CIVI and oxaliplatin $85 \mathrm{mg} / \mathrm{m}^{2}$ ) was then administered at our center every 2 weeks for 3 months. The patient was under active surveillance for 3 years when imaging showed a new $17-\mathrm{mm}$ right lower lobe lung nodule, a $6-\mathrm{mm}$ right apical lung nodule and a $32 \times 26-\mathrm{mm}$ mesenteric lymph node mass at the level of the aortic bifurcation. Lung biopsy confirmed metastatic rectal adenocarcinoma. The patient was started on FOLFIRI-bevacizumab $\left(2,400 \mathrm{mg} / \mathrm{m}^{2} 5\right.$-FU CIVI, $200 \mathrm{mg} / \mathrm{m}^{2}$ $5-\mathrm{FU}, 180 \mathrm{mg} / \mathrm{m}^{2}$ irinotecan and $5 \mathrm{mg} / \mathrm{kg}$ bevacizumab). Irinotecan was discontinued after 2 years due to a parastomal abscess and thrombocytopenia. This regimen was continued until the patient was found to have oligoprogression in the lungs with a progressing single lung metastasis measuring $21 \times 19 \mathrm{~mm}$, and multiple other $<10 \mathrm{~mm}$ metastases throughout each lung, which were stable.

SBRT to the large lung lesion was delivered 6 years after diagnosis at a total dose of $50 \mathrm{~Gy}$ in 5 fractions of $10 \mathrm{~Gy}$ (Fig. 3). Chemotherapy was held during SBRT, and then 5 -FU/bevacizumab was resumed. The patient continues to otherwise have stable disease 10 months after SBRT with no change in systemic chemotherapy.
Patient 3 . The patient was a 47-year-old female who was initially diagnosed with metastatic adenocarcinoma of the colon, with biopsy-proven liver metastases and image-diagnosed bilateral lung involvement, 15 months prior to the first course of SBRT. A left hemicolectomy was performed with $3 / 15$ positive lymph nodes and KRAS proto-oncogene, GTPase mutation. The patient was started on chemotherapy with mFOLFOX6 (similar to patient 1) and $5 \mathrm{mg} / \mathrm{kg}$ bevacizumab. Restaging imaging after 10 months showed a progressive liver metastasis. Systemic chemotherapy was changed to FOLFIRI $\left(135 \mathrm{mg} / \mathrm{m}^{2}\right.$ irinotecan, $400 \mathrm{mg} / \mathrm{m}^{2} 5$-FU IV bolus and $2,400 \mathrm{mg} / \mathrm{m}^{2} 5$-FU CIVI) with $4 \mathrm{mg} / \mathrm{kg}$ ziv-aflibercept every 3 weeks for 3 cycles. Follow-up imaging showed disease progression in the right hilum.

SBRT was delivered to the lung metastasis in the right hilum consisting of a total dose of $30 \mathrm{~Gy}$ in 5 fractions of $6 \mathrm{~Gy}$ (Fig. 4). A repeat positron emission tomography (PET)/CT scan 1 month after SBRT showed mild progression of the multiple liver and lung metastases, and the patient was restarted on FOLFIRI/ziv-aflibercept. The disease responded well with the carcinoembryonic antigen level decreasing from 450 to $75 \mathrm{ng} / \mathrm{ml}$. The patient was maintained on this regimen with surveillance for 1 year when repeat imaging showed progression in a single pulmonary nodule. The case was discussed at the GI TB and the recommendation was to continue the same systemic chemotherapy regimen and pursue SBRT to the left hilum.

SBRT was delivered to the left lung hilum metastasis at 13 months after the first course using a total of $30 \mathrm{~Gy}$ in 5 fractions of 6 Gy (Fig. 4). Following SBRT, the current chemotherapy regimen was re-initiated.

At 16 months after the first course and 3 months after the second course of SBRT, the patient was enrolled in a clinical trial due to disease progression. The systemic disease continues to progress despite additional changes to the treatment plan.

Patient 4 . The patient was a 60 -year-old male diagnosed with stage I (T2NOM0) adenocarcinoma of the rectum 5 years prior to SBRT. The patient was treated initially with $825 \mathrm{mg} / \mathrm{m}^{2}$ neoadjuvant capecitabine twice daily and concurrent radiation therapy to $50.4 \mathrm{~Gy}$ in 1.8-Gy fractions. A transanal resection was performed and the pathological findings were consistent 
A
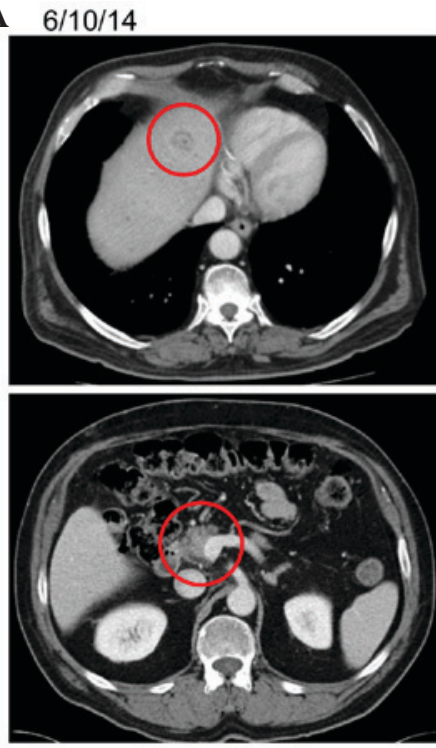

B
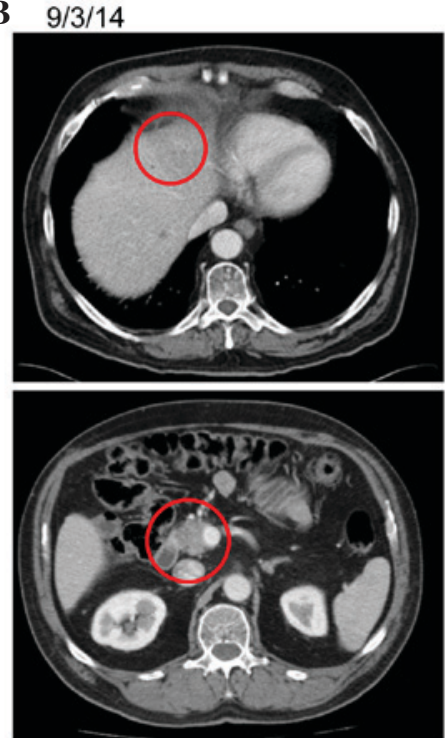

C
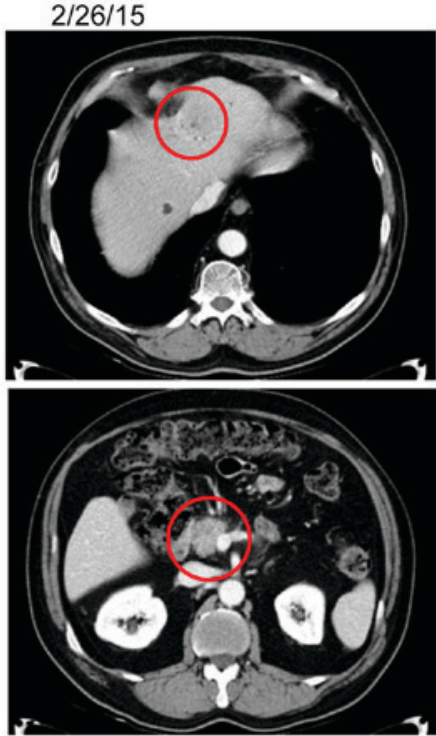

Figure 2. Representative imaging for patient 1. (A) A computed tomography (CT) image of the untreated primary and metastatic disease prior to stereotactic body radiotherapy (SBRT). (B) A CT image of the untreated primary and metastatic disease 3 months after SBRT. (C) A CT image of the untreated primary and metastatic disease 9 months after SBRT.

A

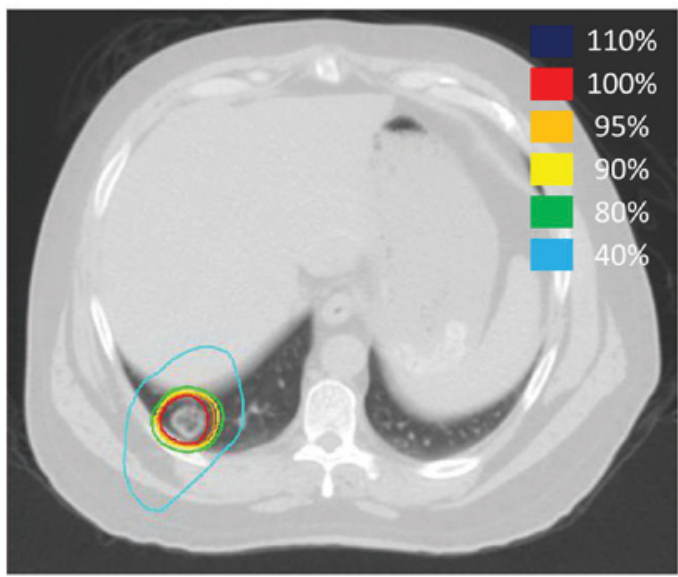

B

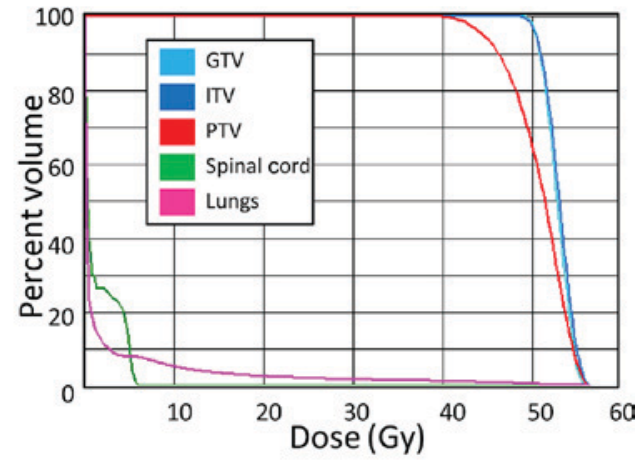

Figure 3. Stereotactic body radiotherapy (SBRT) treatment plan for patient 2. (A) A computed tomography (CT) image of the treatment plan with isodose volumes shown. (B) A dose-volume histogram of the SBRT plan with pertinent structures shown. GTV, gross tumor volume; ITV, internal target volume; PTV, planning target volume.

with adenocarcinoma. Surveillance was continued for 1 year when 4 liver metastases were found. Chemotherapy with mFOLFOX6-bevacizumab $\left(400 \mathrm{mg} / \mathrm{m}^{2} 5\right.$-FU D1, 2,400 mg/m 5 -FU CIVI, $85 \mathrm{mg} / \mathrm{m}^{2}$ oxaliplatin and $5 \mathrm{mg} / \mathrm{kg}$ bevacizumab) was initiated with a good response on follow-up imaging. After 11 months, a right partial hepatectomy and left wedge resection were performed for isolated recurrence, which was followed by a 14-month disease-free period on surveillance imaging.

The patient was then found to have a new liver metastasis, which was also resected. Surveillance was continued for 8 months when PET/CT showed a thickened rectal wall and enlarged perirectal lymph node, as well as a pericardial lymph node that had increased in size since a prior exam. His case was presented at the GI TB and the consensus was for mediastinal biopsy and endoscopic rectal ultrasound for the recurrent disease at the primary site. Following a mediastinal biopsy confirming adenocarcinoma, the patient was started on systemic chemotherapy with $200 \mathrm{mg} / \mathrm{m}^{2}$ oral capecitabine administered twice daily, plus $7.5 \mathrm{mg} / \mathrm{kg}$ intravenous bevacizumab every day for 21 days. The patient was maintained on this regimen with stable disease on follow-up imaging for 11 months when CT imaging indicated progression of the anterior mediastinal nodal disease.

SBRT was delivered at a total dose of $50 \mathrm{~Gy}$ in 10 fractions of $5 \mathrm{~Gy}$ (Fig. 5). Systemic chemotherapy was continued with continued control of the disease for 11 months. Several lung nodules then developed, each $<1 \mathrm{~cm}$ on CT imaging. SBRT to 5 progressive lung metastases was performed to a total of 50 Gy in 10 fractions of 5 Gy (Fig. 5). Following the second round of SBRT, the patient was continued on chemotherapy with serial imaging for 15 months. CT imaging indicated worsening pulmonary metastases with a large right pleural effusion and progression of the anterior mediastinal nodal metastasis. 

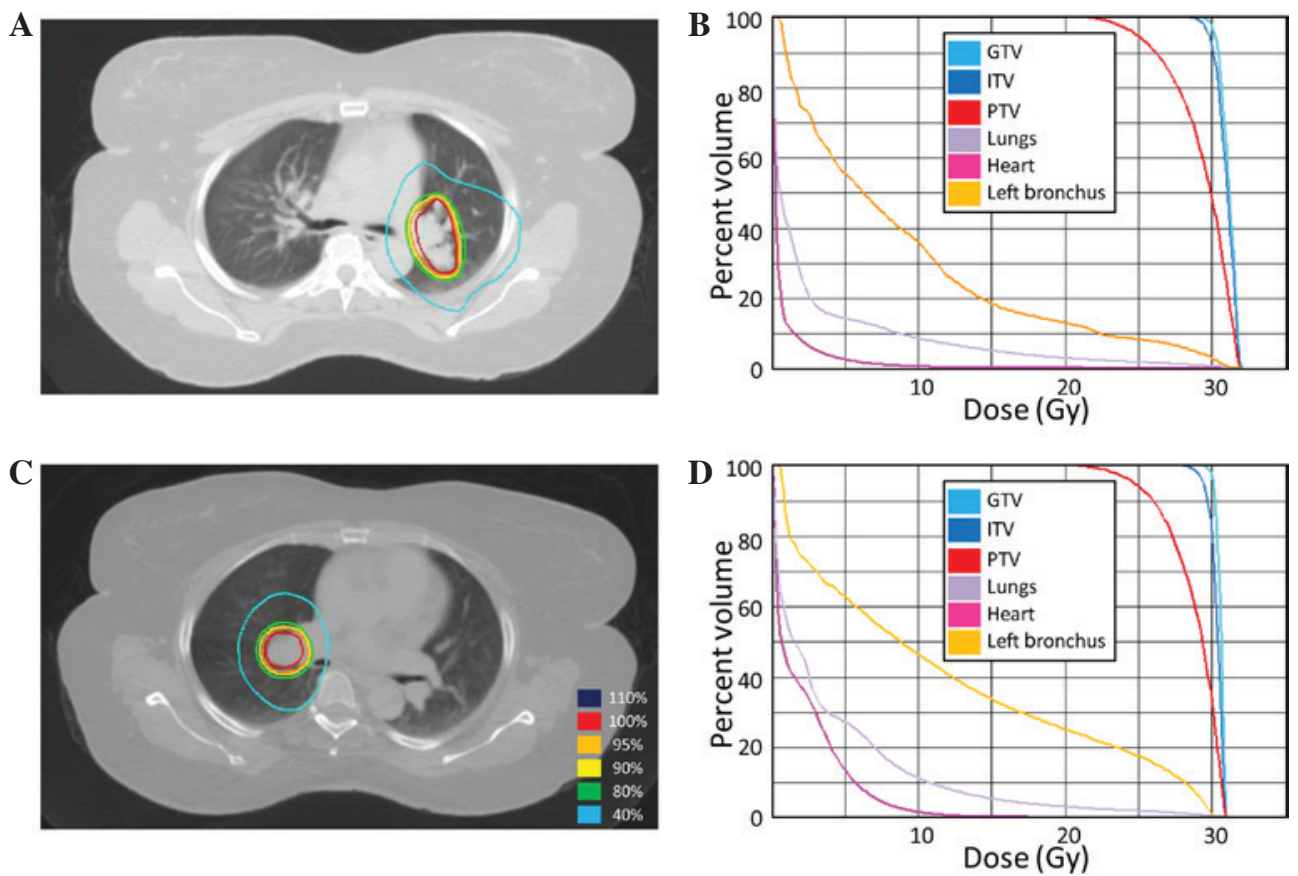

Figure 4. Stereotactic body radiotherapy (SBRT) treatment plans for patient 3. (A) SBRT course 1: A computed tomography (CT) image of the treatment plan with isodose volumes shown. (B) SBRT course 2: A dose-volume histogram of the SBRT plan with pertinent structures shown. (C) SBRT course 2: A CT image of the treatment plan with isodose volumes shown. (D) SBRT course 2: A dose-volume histogram of the SBRT plan with pertinent structures shown. GTV, gross tumor volume; ITV, internal target volume; PTV, planning target volume.
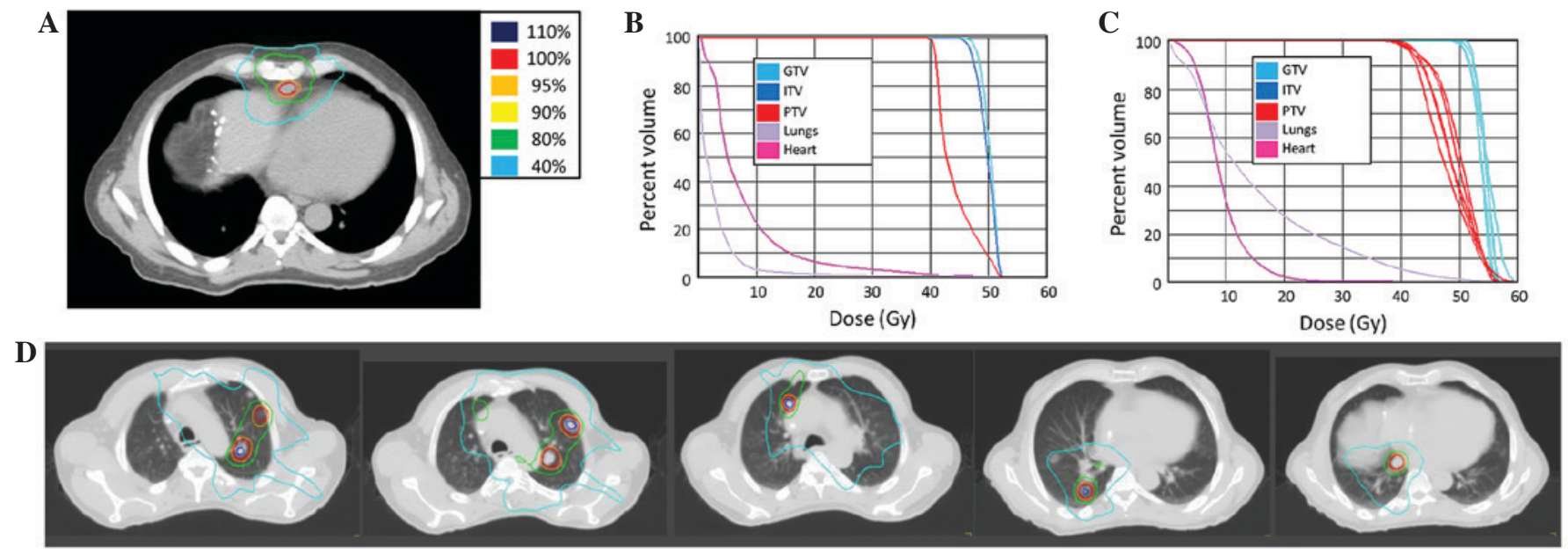

Figure 5. Stereotactic body radiotherapy (SBRT) treatment plans for patient 3. (A) SBRT course 1. A computed tomography (CT) image of the treatment plan with isodose volumes shown. (B) SBRT course 2. A dose-volume histogram of the SBRT plan with pertinent structures shown. (C) SBRT course 2. A combined dose-volume histogram of the SBRT plan with pertinent structures shown. All GTVs and PTVs are shown. (D) SBRT course 2. A CT image of the treatment plan, including all treated metastases, with isodose volumes shown. GTV, gross tumor volume; ITV, internal target volume; PTV, planning target volume.

The systemic chemotherapy was changed 18 months after the first round of SBRT to single-agent panitumumab $(6 \mathrm{mg} / \mathrm{kg}$ every 2 weeks), with irinotecan $\left(180 \mathrm{mg} / \mathrm{m}^{2}\right)$ subsequently added due to continued progression. The patient was subjected to other treatments, and succumbed to progressive systemic disease 2 years and 2 months from the first SBRT.

Patient 5 . The patient was a 68-year-old male who was originally diagnosed with a stage IIIC (pT3N2bM0) proximal transverse colon adenocarcinoma 3 years prior to SBRT. Staging imaging was notable for subcentimeter pulmonary nodules, but no definitive evidence of metastatic disease. The patient underwent a laparoscopic right colectomy followed by adjuvant systemic chemotherapy with standard-dose mFOLFOX-6 $\left(85 \mathrm{mg} / \mathrm{m}^{2}\right.$ oxaliplatin, $400 \mathrm{mg} / \mathrm{m}^{2}$ leucovorin, $400 \mathrm{mg} / \mathrm{m}^{2}$ 5 -FU bolus and $2,400 \mathrm{mg} / \mathrm{m}^{2} 5$-FU over $46 \mathrm{~h}$ ) repeated every 2 weeks. Although several dose reductions were required, there was no evidence of disease progression on follow-up imaging. After a year, surveillance imaging demonstrated two enlarging mesenteric soft-tissue nodules. Chemotherapy was changed to FOLFIRI-bevacizumab $\left(280 \mathrm{mg} / \mathrm{m}^{2} 5-\mathrm{FU}, 2,400 \mathrm{mg} / \mathrm{m}^{2}\right.$ $5-\mathrm{FU}, 180 \mathrm{mg} / \mathrm{m}^{2}$ irinotecan and $5 \mathrm{mg} / \mathrm{kg}$ bevacizumab), with a $50 \%$ dose reduction of the 5-FU bolus. The patient had two breaks from chemotherapy due to non-cancer-related disease 

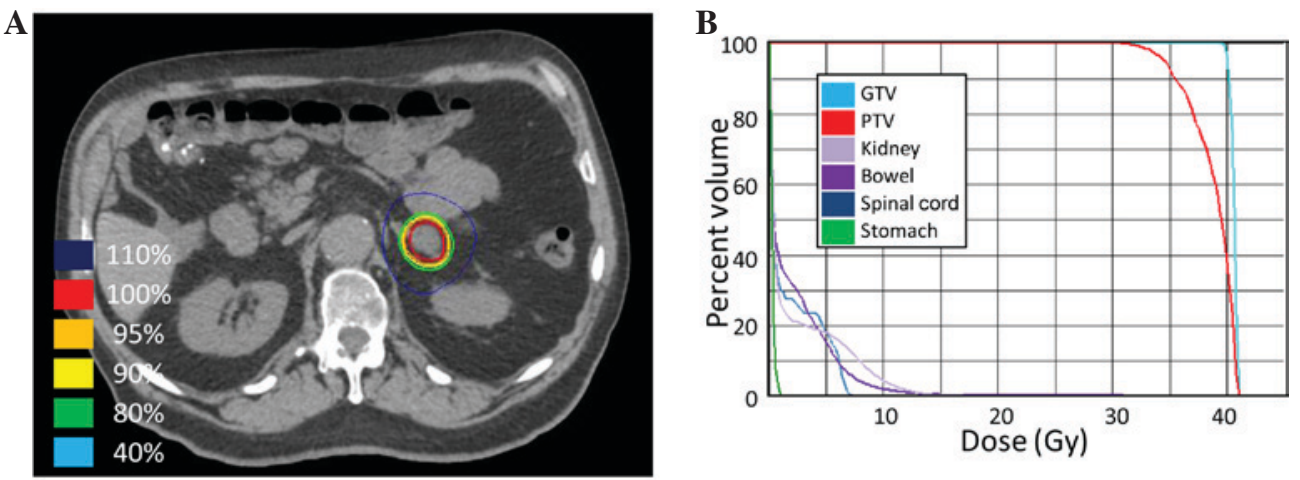

Figure 6. Steretactic body radiotherapy (SBRT) treatment plan for patient 5. (A) A computed tomography (CT) image of the treatment plan with isodose volumes shown. (B) A dose-volume histogram of the SBRT plan with pertinent structures shown. GTV, gross tumor volume; ITV, internal target volume; PTV, planning target volume.

requiring vascular surgery and subsequent complications. After 2 years, repeat imaging revealed interval increase in size of a soft tissue mass in the mesentery with stable lung metastases, and FOLFIRI with bevacizumab was restarted without a 5 -FU bolus $\left(180 \mathrm{mg} / \mathrm{m}^{2}\right.$ irinotecan, $2,400 \mathrm{mg} / \mathrm{m}^{2} 5$-FU CIVI and $5 \mathrm{mg} / \mathrm{kg}$ bevacizumab) with interval response consisting of stable imaging. After 4 months, surveillance imaging showed a new left adrenal nodule, which was discussed during GI TB. The consensus recommendation was for short-interval surveillance. Follow-up imaging 3 months later revealed a slight increase in the left adrenal nodule.

SBRT was delivered at a total of $40 \mathrm{~Gy}$ in 5 fractions of 8 Gy (Fig. 6) to the left adrenal metastasis. Chemotherapy was continued during SBRT. Ziv-alfibercept $(4 \mathrm{mg} / \mathrm{kg}$ ) replaced bevacizumab with FOLFIRI 4 months after completing the SBRT due to systemic disease progression outside of the radiation field.

\section{Discussion}

The present study describes the cases of 5 patients with metastatic cancer treated with SBRT for oligoprogressive disease with the goal of delaying the requirement to transition to subsequent lines of chemotherapy. This goal was achieved in 4 out of 5 patients, each of whom was able to remain on pre-SBRT systemic therapy beyond 7 months without progression warranting a change in chemotherapy. SBRT was well-tolerated and there were no grade 3 or higher toxicities from SBRT, enabling continuation of the same systemic chemotherapy with sustained quality of life. Ongoing disease control is being performed with the same systemic regimen in 2 of the patients, and 1 patient has remained off chemotherapy for 8 months. Additionally, 2 of the patients developed disease progression after 16 and 18 months, 1 of whom has since succumbed. Furthermore, 1 patient was found to have widespread disease progression at the 3-month follow-up imaging.

Biologically, this approach appears to be intuitive since treatment resistance results from cancer adaptations and mutations, which induce subsequent clonal selection (10). However, systemic treatment changes to account for local clonal evolution creates new challenges. Systemic therapeutic options are finite and further mutations accumulate after each line of therapy, as new selection forces are applied even to clones that previously were static and responding to previous treatment. By eradicating resistant, oligoprogressive sub-clones, prematurely discontinuing otherwise effective systemic therapy may be avoided. Significant clinical benefit may be obtained if the duration of each of line of systemic therapy is extended prior to changing to a new regimen. Historically, surgical debulking has been the only way to provide durable disease control for oligometastatic disease, but for a number of patients this carries some morbidity and requires recovery time off of systemic therapy, which allows progression of otherwise stable metastatic disease. Additionally, the surgical recovery of hepatic metastasectomy may stimulate otherwise stable growth factors potentially accelerating disease progression. In the modern era, SBRT has become a novel approach to achieve non-invasive debulking or ablation and has been reported in patients treated with targeted therapy to control their widely metastatic disease (1,11-13).

In a retrospective series (sequential publications including the same patients), Gan et al (11) and Weickhardt et al (1) described the cases of 33 patients administered crizotinib for metastatic non-small cell lung cancer with anaplastic lymphoma kinase-positive tumors. In total, 14 patients exhibited extracranial progression in 4 or fewer tumor sites that were treated with SBRT, yielding excellent local control rates and an increased time to widespread progression. Those patients who were able to continue taking crizotinib for over 12 months experienced significantly higher overall survival times.

Straka et al (13) described the case of a patient with metastatic renal cell carcinoma who developed a solitary area of progression in an adrenal metastasis whilst being administered sunitinib. Rather than switching to a different systemic therapy, SBRT was used to treat the progressing tumor. Sunitinib was then be administered for another 8 months prior to the occurrence of more widespread progression. A Canadian, multi-institutional, single-arm, prospective, phase 2 trial (OZM-053; clinicaltrials.gov identifier NCT02019576) is currently being conducted to study the use of SBRT in metastatic renal cell carcinoma patients who develop oligoprogression whilst being administered first-line sunitinib therapy. SBRT is being used to treat $\leq 5$ progressing tumors, with $\leq 3$ progressing soft-tissue metastases.

Finally, Iyengar et al (12) recently published a prospective phase II trial employing SBRT in patients on second-line 
erlotinib to debulk progressive metastatic disease. This study is subtly different compared with the others in that every site of metastatic disease was treated with SBRT prior to initiation of erlotinib after the patients failed first-line chemotherapy. Nonetheless, an increase in progression-free (14.7 months) and overall (20.4 months) survival times was shown compared with historical benchmarks.

All these studies employed targeted biologically active tyrosine kinase inhibitors, but to the best of our knowledge, no previously published study has indicated that this approach would be beneficial in patients on systemic chemotherapy. Iyengar et al (12) used SBRT following systemic treatment failure, but did not continue the current regimen; they instead initiated a targeted agent once debulking was accomplished.

There are several limitations of the present series, including the small patient population treated in this manner and the lack of a comparison group. Despite these limitations, this study provides proof of principle and clinical rationale for considering the thoughtful use of SBRT for treatment of oligoprogressive disease in select situations where continuation of an otherwise effective systemic chemotherapy is reasonable.

One of the greatest strengths of the present study is the novel approach to evaluating the efficacy of the treatment by measuring the time from the treatment with SBRT to the change in chemotherapy. This is an innovative strategy to evaluate progressive metastatic disease treatment efficacy and we advocate for systemic change in this arena. Future studies will benefit from this approach, as patients in the metastatic setting often experience short overall survival times and the intent of the treatment is to extend quality of life, which may be difficult to prove significant, since it is difficult to objectively measure. Furthermore, once patients enroll in a hospice and decide to discontinue therapy, the endpoint of overall survival is no longer relevant. Other endpoints such as health care resource utilization, pharmacoeconomic analyses and patient-reported quality of life should be considered as correlative endpoints in future studies.

To summarize, the current study presents the cases of 5 patients who achieved an extended, high quality of life following SBRT delivered to increase the duration of effective systemic chemotherapy, with no significant toxicity. We believe that this strategy will improve the duration of effective chemotherapy and allow for increased, high quality longevity in a select group of patients with oligoprogression.

\section{References}

1. Weickhardt AJ, Scheier B, Burke JM, Gan G, Lu X, Bunn PA Jr, Aisner DL, Gaspar LE, Kavanagh BD, Doebele RC and Camidge DR: Local ablative therapy of oligoprogressive disease prolongs disease control by tyrosine kinase inhibitors in oncogene-addicted non-small-cell lung cancer. J Thorac Oncol 7: 1807-1814, 2012.

2. Gerlinger M, Rowan AJ, Horswell S, Larkin J, Endesfelder D, Gronroos E, Martinez P, Matthews N, Stewart A, Tarpey P, et al: Intratumor heterogeneity and branched evolution revealed by multiregion sequencing. N Engl J Med 366: 883-892, 2012.

3. Ding L, Ellis MJ, Li S, Larson DE, Chen K, Wallis JW, Harris CC, McLellan MD, Fulton RS, Fulton LL, et al: Genome remodelling in a basal-like breast cancer metastasis and xenograft. Nature 464: 999-1005, 2010.

4. Yachida S, Jones S, Bozic I, Antal T, Leary R, Fu B, Kamiyama M, Hruban RH, Eshleman JR, Nowak MA, et al: Distant metastasis occurs late during the genetic evolution of pancreatic cancer. Nature 467: 1114-1117, 2010.

5. Wilson TR, Longley DB and Johnston PG: Chemoresistance in solid tumours. Ann Oncol 17 (Suppl 10): x315-x324, 2006.

6. Milano MT, Katz AW, Zhang H and Okunieff P: Oligometastases treated with stereotactic body radiotherapy: Long-term follow-up of prospective study. Int J Radiat Oncol Biol Phys 83: 878-886, 2012.

7. Takeda A, Sanuki N, Tsurugai Y, Oku Y and Aoki Y: Stereotactic body radiotherapy for patients with oligometastases from colorectal cancer: Risk-adapted dose prescription with a maximum dose of 83-100 Gy in five fractions. J Radiat Res: March 16, 2016 (Epub ahead of print).

8. Filippi AR, Guerrera F, Badellino S, Ceccarelli M, Castiglione A, Guarneri A, Spadi R, Racca P, Ciccone G, Ricardi U and Ruffini E: Exploratory analysis on overall survival after either surgery or stereotactic radiotherapy for lung oligometastases from colorectal cancer. Clin Oncol (R Coll Radiol) 28: 505-512, 2016.

9. Edge S, Byrd DR, Compton CC, Fritz AG, Greene FL and Trotti A (eds): American Joint Committee on Cancer. AJCC Cancer Staging Manual. 8th edition. Springer, New York, NY, 2010.

10. Talmadge JE: Clonal selection of metastasis within the life history of a tumor. Cancer Res 67: 11471-11475, 2007.

11. Gan GN, Weick hardt AJ, Scheier B, Doebele RC, Gaspar LE, Kavanagh BD and Camidge DR: Stereotactic radiation therapy can safely and durably control sites of extra-central nervous system oligoprogressive disease in anaplastic lymphoma kinase-positive lung cancer patients receiving crizotinib. Int J Radiat Oncol Biol Phys 88: 892-898, 2014.

12. Iyengar P, Kavanagh BD, Wardak Z, Smith I, Ahn C, Gerber DE, Dowell J, Hughes R, Abdulrahman R, Camidge DR, et al: Phase II trial of stereotactic body radiation therapy combined with erlotinib for patients with limited but progressive metastatic non-small-cell lung cancer. J Clin Oncol 32: 3824-3830, 2014.

13. Straka C, Kim DW, Timmerman RD, Pedrosa I, Jacobs C and Brugarolas J: Ablation of a site of progression with stereotactic body radiation therapy extends sunitinib treatment from 14 to 22 months. J Clin Oncol 31: e401-e403, 2013. 